\title{
On the Radial Structure of Radiatively Inefficient Accretion Flows with Convection
}

\section{Citation}

Abramowicz, Marek A., Igor V. Igumenshchev, Eliot Quataert, and Ramesh Narayan. 2002. "On the Radial Structure of Radiatively Inefficient Accretion Flows with Convection." The Astrophysical Journal 565 (2): 1101-6. https://doi.org/10.1086/324717.

\section{Permanent link}

http://nrs.harvard.edu/urn-3:HUL.InstRepos:41384944

\section{Terms of Use}

This article was downloaded from Harvard University's DASH repository, and is made available under the terms and conditions applicable to Other Posted Material, as set forth at http:// nrs.harvard.edu/urn-3:HUL.InstRepos:dash.current.terms-of-use\#LAA

\section{Share Your Story}

The Harvard community has made this article openly available.

Please share how this access benefits you. Submit a story.

Accessibility 


\title{
On the Radial Structure of Radiatively Inefficient Accretion Flows with Convection
}

\author{
Marek A. Abramowicz ${ }^{1}$, Igor V. Igumenshchev ${ }^{2,3}$, Eliot Quataert ${ }^{4}$ and Ramesh Narayan \\ Harvard-Smithsonian Center for Astrophysics, 60 Garden Street, Cambridge MA 02138
}

\begin{abstract}
We consider the radial structure of radiatively inefficient hydrodynamic accretion flows around black holes. We show that low-viscosity flows consist of two zones: an outer convection-dominated zone and an inner advection-dominated zone. The transition between these two zones occurs at $\sim 50$ Schwarzschild radii.
\end{abstract}

Subject headings: accretion, accretion disks — black hole physics — convection

\footnotetext{
${ }^{1}$ Department of Astronomy \& Astrophysics, Göteborg University \& Chalmers University of Technology, 412-96 Göteborg, Sweden

${ }^{2}$ Laboratory for Laser Energetics, University of Rochester, 250 East River Road, Rochester NY 14623-1299.

${ }^{3}$ Institute of Astronomy, 48 Pyatnitskaya Ulitsa, 109017 Moscow, Russia.

${ }^{4}$ Institute for Advanced Study, Princeton NJ 08540; Chandra Fellow
} 


\section{Introduction}

Observations of low luminosity black holes in galactic nuclei and X-ray binaries have been modeled within the framework of a radiatively inefficient accretion flow (RIAF). In these models, radiative losses are small because of the low particle density of the accreting plasma at low mass accretion rates (see Narayan, Mahadevan \& Quataert 1998 and Kato, Fukue \& Mineshige 1998 for reviews).

Hydrodynamical simulations of low-viscosity RIAFs in two dimensions (Igumenshchev \& Abramowicz 1999, 2000; Stone, Pringle \& Begelman 1999) and three dimensions (Igumenshchev, Abramowicz \& Narayan 2000) have shown that these flows are convectively unstable (see also Narayan \& Yi 1994, 1995), and that the convection has a significant effect on the structure of the flow. Narayan, Igumenshchev \& Abramowicz (2000, hereafter NIA) and Quataert \& Gruzinov (2000, hereafter QG) constructed selfsimilar solutions for convection-dominated accretion flows (CDAFs), and used these to explore the relevant physics and to explain the results of the numerical simulations.

In this paper we show via analytical arguments that the radial structure of a low-viscosity RIAF consists of two distinct zones: an outer convectiondominated zone and an inner advection-dominated zone $(\S 2)$. We present global solutions of an idealized height-integrated set of equations ( $\S 3$ ) and show that the radial structure of the advection-dominated zone differs significantly from the canonical self-similar solution for an advection-dominated accretion flow (ADAF, Narayan \& Yi 1994, 1995). This is because of the strong influence of the boundary conditions. We then compare the theoretical results with numerical simulations ( $(4)$ and find that there is good agreement. In particular, both the global solutions and the numerical simulations show that the transition from the inner ADAF zone to the outer CDAF zone occurs at a radius $R_{A C} \sim 50 R_{g}$, where $R_{g}=2 G M / c^{2}$ is the gravitational radius of the accreting black hole of mass $M$.

\section{Analysis of Self-Similar CDAFs}

We consider a rotating accretion flow with a selfsimilar scaling of the height-averaged angular velocity $\Omega$ and isothermal sound speed $c_{s}$, and the scale height $H$ :

$$
\Omega(R)=\Omega_{0} \Omega_{K} \propto R^{-3 / 2},
$$

$$
\begin{aligned}
& c_{s}^{2}(R)=c_{0}^{2} v_{K}^{2} \propto R^{-1}, \\
& H(R)=c_{s} / \Omega_{K}=c_{0} R,
\end{aligned}
$$

where $\Omega_{K}=\sqrt{G M / R^{3}} \equiv v_{K} / R$ is the Keplerian angular frequency at radius $R$, and $\Omega_{0}$ and $c_{0}$ are dimensionless constants. We consider a power-law radial dependence for the height averaged density,

$$
\rho(R) \propto R^{-a},
$$

which corresponds to a radial velocity varying as

$$
v_{R}(R)=\dot{M} / 4 \pi R H \rho \propto R^{a-2},
$$

where $\dot{M}$ is the mass accretion rate. The index $a$ is equal to $1 / 2$ for a self-similar CDAF (NIA) and $3 / 2$ for a self-similar ADAF (Gilham 1981; Begelman \& Meier 1982; Narayan \& Yi 1994, 1995). We refer the reader to NIA for the calculation of the constants $\Omega_{0}$ and $c_{0}$.

Here we focus on the consistency of the self-similar CDAF solution. For this purpose we consider the internal energy equation describing a RIAF,

$$
\rho v_{R} T \frac{d s}{d R}+\frac{1}{R^{2}} \frac{d}{d R}\left(R^{2} F_{c}\right)=Q^{+}-Q_{r a d},
$$

where $s$ is the specific entropy, $F_{c}$ is the outwardly directed convective energy flux, $Q^{+}$is the rate of energy dissipation per unit volume, and $Q_{\text {rad }}$ is the rate of energy loss per unit volume through radiation. By definition, radiative losses are small in a RIAF, so we neglect $Q_{\text {rad }}$ in equation (4). The first term on the left hand side of equation (4) describes the inward advection of the internal energy. For the scaling laws given in (1) and (2) this advection term takes the form,

$$
\rho v_{R} T \frac{d s}{d R}=\frac{\dot{M} c^{2}}{4 \pi R H} \frac{c_{0}^{2}}{2} \frac{R_{g}}{R^{2}}\left(\frac{1}{\gamma-1}-a\right),
$$

where $c$ is the speed of light and $\gamma$ is the adiabatic index of the accreting gas. For a rotating accretion flow, the energy dissipation rate $Q^{+}$can be written as

$$
Q^{+}=-\left(J_{v}+J_{c}\right) \frac{d \Omega}{d R},
$$

where $J_{v}$ and $J_{c}$ are the angular momentum fluxes due to viscosity and convective motions, respectively. In the case of an $\operatorname{ADAF}(a=3 / 2)$, convection is ignored and we set $J_{c}=F_{c}=0$. Since viscosity transports angular momentum outwards, i.e. $J_{v}>0$, the dissipation rate $Q^{+}$is a positive quantity, and in equation (4), $Q^{+}$balances the advection term (5). 
The situation is very different in a CDAF. NIA considered a self-similar CDAF in the limit of a nonaccreting "convective envelope", in which $v_{R}=0$ and the advection term (5) vanishes. How can a viscous differentially-rotating fluid configuration not accrete? This is possible because, as demonstrated explicitly via three-dimensional numerical simulations (Igumenshchev et al. 2000) and earlier through analytic methods (Ryu \& Goodman 1992; Stone \& Balbus 1996), axisymmetric convection transports angular momentum inwards, i.e. $J_{c}<0$, whereas viscosity transports it outwards. Thus, by ensuring that convection and viscosity cancel each other exactly, i.e. $J_{v}+J_{c}=0$, the convective envelope is able to achieve a configuration with no net angular momentum transport and hence no mass accretion. Since $J_{v}+J_{c}=0$, an additional consequence is that $Q^{+}=0$ (see eq. [6]). The energy equation (4) then has only one term left, namely the divergence of the convective flux, and the only way to satisfy this equation is for the divergence of the flux to vanish, i.e. to have $F_{c}(R) \propto R^{-2}$.

The non-zero outward energy flux $F_{c}$ corresponds to a "convective luminosity", $L_{c}=4 \pi R H F_{c}$, which is independent of radius. The source of this luminosity is not specified in the self-similar solution and is formally located at $R=0$. NIA argued that in the case of a real CDAF there would be a small but non-zero accretion rate, $\dot{M} \neq 0$, and the convective luminosity would be supplied by the gravitational release of binding energy in the innermost region of the accretion flow. Thus, we may write

$$
L_{c}=\varepsilon \dot{M} c^{2},
$$

where $\varepsilon$ is the "convective efficiency". A non-zero accretion rate requires a non-zero net angular momentum flux directed outward, $J_{v}+J_{c}>0$, so according to (6) one would also have $Q^{+}>0.5$

We now estimate the importance of different terms in the energy equation (4). For this we integrate equation (4) over a spherical volume from $R$ to infinity. After the integration, the terms which correspond to the advection term (5) and the dissipation term (6) both scale $\propto R^{-1}$, whereas the term describing the convective energy transport is equal to $L_{c}=$ constant. Thus, convective energy transport dominates the physics at large radii, and the self-similar CDAF

\footnotetext{
${ }^{5}$ Taking into account that the release of gravitational and rotational energy is the ultimate source of any dissipation in the accretion flow, one can conclude $Q^{+} \propto(G M / R) \rho v_{R} / R \propto R^{-4}$.
}

solution is a good approximation for describing this region of a RIAF. At small radii, however, the advection term and the viscous dissipation term become more important. By comparing the inward advective energy flux,

$\dot{E}_{a d v}=\int_{R}^{\infty} 4 \pi R H \rho v_{R} T \frac{d s}{d R} d R=\frac{c_{0}^{2}}{2}\left(\frac{1}{\gamma-1}-a\right) \frac{R_{g}}{R} \dot{M} c^{2}$,

with the convective luminosity $L_{c}$ (eq. [7]), we may determine the transition radius $R_{A C}$ that separates the outer convection-dominated zone and the inner advection-dominated zone. Using the estimate of $c_{0}^{2}$ from QG or NIA's Appendix, and applying the condition $\dot{E}_{a d v}=L_{c}$, we obtain

$$
\frac{R_{A C}}{R_{g}} \sim \frac{1}{\varepsilon} \frac{3-\gamma}{2(\gamma+3)} .
$$

For a quantitative estimate of $R_{A C}$ we need to determine the value of the convective efficiency parameter $\varepsilon$. Since it is hard to determine this from first principles, we must either solve for $\varepsilon$ as an eigenvalue of the global problem ( $\S 3)$ or determine it from numerical simulations $(\S 4)$.

\section{Global CDAFs}

In this section we present a sample height integrated global CDAF model which satisfies proper inner and outer boundary conditions. This is the CDAF analogue of the global ADAF models calculated by Narayan, Kato, \& Honma (1997) and Chen, Abramowicz, \& Lasota (1997). The solution sheds further light on the CDAF-ADAF transition discussed in $\S 2$.

In the absence of radiation, the conservation of mass, angular momentum, and total energy can be expressed via conserved fluxes:

$$
\begin{gathered}
\dot{M}=-4 \pi R H \rho v_{R}=\text { const } \\
4 \pi F_{L}=-\dot{M} R^{2} \Omega+4 \pi R H\left(J_{v}+J_{c}\right)=\text { const, } \\
4 \pi F_{E}=-\dot{M} B+4 \pi R H \Omega\left(J_{v}+J_{c}\right)+4 \pi R H F_{c}=\text { const }
\end{gathered}
$$

where $B=0.5 v_{R}^{2}+\phi+0.5 R^{2} \Omega^{2}+\gamma c_{s}^{2} /(\gamma-1)$ is the Bernoulli function, and we take the Paczyński \& Wiita (1980) form of the gravitational potential, $\phi=$ $-G M /\left(R-R_{g}\right)$, to mimic a Schwarzschild black hole. Note that the radial derivative of equation (12) yields 
equation (4) with $Q_{\text {rad }}=0$. In addition to equations (10)-(12), we need the radial momentum equation:

$$
v_{R} \frac{d v_{R}}{d R}=R \Omega^{2}-\frac{d \phi}{d R}-\frac{1}{\rho} \frac{d p}{d R}
$$

where $p=\rho c_{s}^{2}$ is the pressure.

We use the expressions for $J_{v}, J_{c}$ and $F_{c}$ from NIA's Appendix except that we employ a causal prescription for the convective transport of energy and angular momentum. [ $^{6}$ We take the convective diffusion coefficient to be $\propto\left(1-v_{R}^{2} / c_{c}^{2}\right)^{2}$ for $\left|v_{R}\right|<c_{c}$ and 0 otherwise (cf. Popham \& Narayan 1992; Narayan 1992), where we choose $c_{c}=\alpha c_{s}$ (i.e. roughly the convective velocity). The condition $\left|v_{R}\right|=c_{c}$ defines a transition radius, $R_{A C}$, inside of which there is no convective transport of angular momentum and energy. For $R<R_{A C}$, the inflow velocity of the gas exceeds the characteristic convective velocity, and convective transport is less important than bulk advective transport (the convective transport vanishes in our simplified model). Outside of $R_{A C}$ the inflow velocity of the gas is less than the characteristic convective velocity and the flow is roughly "static", as in the self-similar CDAF solution.

We note that $J_{v}$ depends on the value of the Shakura-Sunyaev viscosity parameter $\alpha$, so the global solution depends on the choice we make for this parameter. Other parameters are the adiabatic index $\gamma$ and the outer radius of the accretion flow $R_{\text {out }}$. In addition, although the causal prescription for convective transport is well motivated (e.g., Narayan 1992), the proper cutoff velocity (i.e., $c_{c}$ ) is somewhat uncertain.

Equations (10)-(13) are four first order differential equations. Since we are interested in solutions in which the gas goes through a sonic point on its way into the black hole, the sonic radius $R_{s}$ is an additional unknown which needs to be determined. The 3 conserved fluxes, $\dot{M}, F_{L}$, and $F_{E}$ are also eigenvalues determined in a self-consistent manner. This implies that the convective efficiency $\varepsilon$ of equation (7) is an eigenvalue of the global model, rather than a free parameter as in the self-similar solution. The transition radius $R_{A C}$ is a final eigenvalue. For $R<R_{A C}$, $F_{c}=0$ and $J_{c}=0$ because of the causal prescription for convective transport. One can show that the

\footnotetext{
${ }^{6}$ For numerical reasons, we were unable to find global solutions without employing a causal transport prescription. This is somewhat unsatisfying because the causal prescription explicitly imposes an inner ADAF zone on the global CDAF solution.
}

vanishing of $F_{C}$ means that $F_{E}$ and $F_{L}$ are not independent, $F_{L}=\left.F_{E}\left(R^{2} \Omega / B\right)\right|_{R=R_{s}}$. Thus, we require a total of 8 boundary conditions to solve for the 4 variables $\left(\rho, v_{R}, c_{s}, \Omega\right)$ and 4 independent eigenvalues $\left(R_{s}, R_{A C}, \dot{M}, F_{E}\right)$.

At the outer radius of the flow we specify $\rho, c_{s}$, and $\Omega$. The choice of $\rho$ is arbitrary and simply sets the scaling for the density in the problem. We use the self-similar CDAF values for $c_{s}$ and $\Omega$ from QG at the outer boundary. Igumenshchev \& Abramowicz $(1999,2000)$ showed from numerical simulations of RIAFs that strong convection is present only for relatively small values of the viscosity parameter, $\alpha<0.1$. For such values of $\alpha, R_{A C}>R_{s}$. At the sonic point $R=R_{s}$, convection is thus unimportant and the 2 boundary conditions at this radius are identical to those in the global ADAF models of Narayan et al. (1997). We also apply the no-torque condition at the sonic point The final two boundary conditions are at the transition radius $R_{A C}$. We guess a value for $R_{A C}$ and find the solution from the outer boundary to $R_{A C}$ and from $R_{A C}$ to the inner boundary (the sonic point), requiring $\left|v_{R}\right|=c_{c}$ at $R_{A C}$. We then iterate until all flow variables are continuous across $R_{A C}$.

Figure 1 shows the density, radial velocity, and convective velocity $c_{c}$ for a global solution with $\alpha=0.03$ and $\gamma=1.5$. The sonic point of the solution is at $R \approx 2.6 R_{g}$, while the transition radius is located at $R_{A C} \approx 35 R_{g}$. The angular momentum flux in the solution is $4 \pi F_{L} \approx-1.64 \dot{M} R_{g} c$ while the energy flux is $4 \pi F_{E} \approx 0.0045 \dot{M} c^{2}$. At large radii the energy flux is entirely carried by convection, so this corresponds to a convective efficiency of $\varepsilon \approx 0.0045$. For this value of $\varepsilon$ and $\gamma=1.5$, equation (9) predicts $R_{A C}=37 R_{g}$, which is very close to the numerically determined location of the transition radius.

The density profile in Figure 1 is reasonably well described by the $\rho \propto R^{-1 / 2}$ power law of the selfsimilar CDAF solution at all radii. For $R>R_{A C}$ convective transport indeed dominates the energy transport and the scaling $\rho \propto R^{-1 / 2}$ is needed in order to carry a constant convective luminosity outwards (see $\S 2$ and NIA, QG); in this regime the self-similar CDAF solution describes the flow structure quite well.

For $R<R_{A C}$, however, convective transport of energy and angular momentum vanishes in our causal

\footnotetext{
${ }^{7}$ Narayan et al. (1997) applied the no-torque condition close to the black hole horizon; we do not expect this to make a large difference.
} 
prescription. Here, the radial inflow velocity exceeds the convective velocity and bulk (advective) transport of energy dominates. The density profile is nonetheless close to the CDAF scaling of $R^{-1 / 2}$ and much flatter than the $R^{-3 / 2}$ profile of a self-similar ADAF. The reason for this is that the gas is rapidly accelerating toward the sonic point on its way into the black hole. Quite generally, this rapid acceleration leads to a steep radial velocity profile and thus a flat density profile (see, e.g., Narayan et al.'s 1997 and Chen et al.'s 1997 global ADAF models for similar results).

\section{Comparison with Numerical Simulations}

Igumenshchev (2000) and Igumenshchev \& Abramowicz (2000) found that the convective efficiency is $\varepsilon \simeq$ $0.003-0.01$ in two-dimensional simulations of RIAFs, with only a weak dependence on the viscosity parameter $\alpha$ and the adiabatic index $\gamma$. We expect that three-dimensional models of convective RIAFs will show values of $\varepsilon$ in the same range, because of the close qualitative and quantitative similarities between the numerical results in two and three dimensions (Igumenshchev et al. 2000). Taking a characteristic value of $\varepsilon=0.005$, we obtain from equation (9): $R_{A C} \simeq 30 R_{g}$ for $\gamma=5 / 3$ and $R_{A C} \simeq 40 R_{g}$ for $\gamma=4 / 3$. These values for $\epsilon$ and $R_{A C}$ are in good agreement with the values obtained with the global model described in $\S 3$. We now try to estimate $R_{A C}$ directly from the numerical simulations.

The presence of two distinct zones in a RIAF, namely an outer convection-dominated zone and an inner advection-dominated zone, may be demonstrated in a numerical simulation by considering the "mass inflow rate" $\dot{M}_{i n}$ as a function of radius. We define $\dot{M}_{i n}(R)=\int \rho v_{R} d S$, where the surface integral is limited to those gas elements at radius $R$ that have $v_{R}<0$. Because a CDAF is convectively turbulent and accretes slowly, roughly half the mass at any radius at any time will be flowing in and half will be flowing out. This gives $\dot{M}_{i n} \simeq 4 \pi R H \rho \tilde{v} / 2$, where $\tilde{v}$ is the rms velocity of the turbulent eddies. It can be shown that $\tilde{v} \sim \alpha v_{K} \propto R^{-1 / 2}$ (NIA). Therefore, for $\rho \propto R^{-1 / 2}$ and $H \propto R$, one finds that $\dot{M}_{i n} \propto R$. In an ADAF on the other hand, convection is absent and so the mass inflow rate is independent of radius, $\dot{M}_{i n}(R)=$ const.

Figure 2 shows $\dot{M}_{i n}(R)$ (solid line) for a twodimensional simulation of a RIAF with $\alpha=0.01$ and $\gamma=5 / 3$ (the numerical technique is similar to that used by Igumenshchev \& Abramowicz 2000). This simulation was done with the Paczyǹski \& Wiita (1980) pseudo-potential. The plot clearly shows that the accretion flow consists of two different zones: an inner advection-dominated zone, in which $\dot{M}_{i n}=$ const, and an outer convection-dominated zone, in which $\dot{M}_{i n} \propto R$. The transition radius between the two zones is located at $R_{A C} \simeq 50 R_{g}$, which is roughly consistent with the scaling given in equation (9).

Figure 3 shows a snapshot of the velocity streamlines of the particular numerical simulation analyzed in Fig. 2. We see numerous vortices associated with the convective motions in the flow. Interestingly, unlike in the oversimplified global model described in $\S 3$, we see that convective motions are present even in the inner regions of the RIAF $\left(R<R_{A C}\right)$, despite the dominance of advection here. In fact, the convective blobs primarily originate in the advection-dominated part and move outward. According to our estimate (see equation [5] and [7]), the major energy release happens in the advection-dominated part of RIAFs, where the advection and dissipation terms are almost equally important and dominate the convective energy transport. Only a small relative excess of the energy dissipation rate over the inward advection of energy supports turbulence in RIAFs and provides the convective luminosity $L_{c}$. This excess determines the value of the convective efficiency $\varepsilon$.

\section{Discussion and Conclusions}

We have considered the radial structure of radiatively inefficient accretion flows (RIAFs). Based on an analysis of self-similar solutions we conclude that the flow consist of two parts: an outer convectiondominated part where the radial velocity is highly subsonic and an inner advection-dominated part where the gas flows rapidly inwards. The location of the transition radius $R_{A C}$ between these two parts depends primarily on the convective efficiency $\varepsilon$ (see equation [9]). We estimate $\epsilon \approx 0.003-0.01$ and $R_{A C} \sim 50 R_{g}$ directly from numerical simulations (§4). In addition, in 1D steady state global calculations of the structure of RIAFs, which include proper boundary conditions, $\epsilon$ and $R_{A C}$ can be determined as eigenvalues. The global calculations yield $R_{A C} \approx 35 R_{g}$ and $\epsilon \approx 0.0045$ (§3), in good agreement with the simulations.

Convective motions and the associated outward energy flux originate in the inner advection-dominated 
part of the flow. This energy flux supports the turbulent structure of the flow in the outer convectiondominated part. The radial profiles of density and velocity in the inner advection-dominated part of the flow differ significantly from self-similar scalings due to the influence of boundary conditions ( $\S 3)$.

Our present results are based on a viscous hydrodynamical (HD) approach to studying RIAFs. The HD approach is not self-consistent because it requires one to assume the presence of viscosity with an $a$ priori unknown strength. A fully consistent approach can be provided within the framework of magnetohydrodynamics (MHD), in which torques due to magnetic interactions transport angular momentum. It is not presently clear how the concept of CDAFs obtained in the HD approach will be modified after construction of MHD models of RIAFs.

We do not expect significant qualitative differences between HD and MHD models if the gravitational and rotational energies are released locally in the accretion flow, e.g., by magnetic reconnection and/or a turbulent energy cascade. If, however, the free energy is removed from the bulk of the accretion flow via nonlocal interactions, such as the stretching of large-scale magnetic field lines resulting in an efficient Poynting flux, or the formation of MHD driven winds/outflows, one would expect qualitative differences between HD and MHD models. In addition, one would expect a significant modification of the CDAF solution if angular momentum is efficiently removed from the accretion flow by a large-scale magnetic field in a disk corona (contrary to the CDAF model in which angular momentum transport is due to local viscous interactions).

MHD simulations of spherical accretion flows (Igumenshchev \& Narayan 2001) support the idea of local generation of energy via magnetic reconnection, which leads to the development of efficient convection. The structure of these models closely resembles CDAFs. Recent MHD simulations of rotating flows do show unstable motions, which leads to the development of efficient turbulence, but they do not show clear behaviour to either support or contradict the CDAF model. For example, Machida, Matsumoto, \& Mineshige (2001) claim that the structure of their MHD models closely resembles that found in HD CDAFs. However, Hawley, Balbus, \& Stone (2001) stress that their models do not demonstrate convective motions and that the models are instead turbulent due to the magneto-rotational instability in shearing flows. This discrepancy indicates that further development of the MHD models is required.

Recently, Balbus (2001) has discussed the stability criteria for rotating magnetized accretion flows. These criteria replace the Høiland criteria for a plasma with thermal conduction along the magnetic field lines. According to Balbus's criteria a magnetized fluid is unstable even if it is marginally stable to the Høiland criteria. The new criteria have been obtained in the short-wavelength approximation, and it is not straightforward to know how the instability works on larger scales, which might be more important for the structure of an accretion flow. It is also interesting to clarify what Balbus's criteria imply for axisymmetric convection in the presence of a predominantly toroidal magnetic field. Such convection is expected to be present in magnetized CDAFs.

Assuming that the radial structure of RIAFs described in the present paper is basically correct, it is interesting to ask what the implications are for observations of accreting black holes. It has been proposed that for luminosities below a few percent of the Eddington luminosity, accretion flows around black holes switch to a RIAF (cf. Narayan et al. 1998). In the scenario proposed by Narayan (1996) and developed by Esin, McClintock \& Narayan (1997), the accretion flow consists of a radiatively efficient thin disk outside a transition radius $R_{t r}$ and an ADAF inside $R_{t r}$. Spectra computed with such a model agree well with observations of black hole X-ray binaries in the "intermediate state" and "low state" (Esin et al. $1997,1998)$. The models typically require $R_{t r}$ of order $10 R_{g}$ to a few tens of $R_{g}$. In a recent study, Esin et al. (2001) determined that the black hole binary XTE J1118+480 had $R_{t r} \sim 50 R_{g}$ at a time when its X-ray luminosity was $\sim 10^{-3} L_{E d d}$. According to the present paper, an ADAF is a good approximation to the structure of a RIAF so long as $R \lesssim 50 R_{g}$. Therefore, the above models are consistent.

For yet lower luminosities, e.g., black holes in the "quiescent state", the transition radius in models is typically at $R_{t r} \sim 10^{3}-10^{4} R_{g}$, and in some models there is no thin disk at all (see Narayan et al. 1998 for examples). Published models of the quiescent state have generally made use of a pure ADAF (or a spherical Bondi flow, e.g. Melia 1992), whereas our present results suggest that the flow might take the form of a CDAF for $R \gtrsim 50 R_{g}$. How will this affect the predicted spectrum? The principal difference between an ADAF (or a Bondi flow) and a CDAF is the value 
of the index $a$ (cf eq. [2]) in the radial density profile: $a=3 / 2$ for a self-similar ADAF, $a=1 / 2$ for a self-similar CDAF. Quataert \& Narayan (1999) studied models with different values of $a$ and showed that these models can produce similar spectra provided the fraction of viscous heat energy that goes into electrons (which is assumed to be close to zero for an ADAF) is appropriately adjusted. For a CDAF, perhaps as much as half the heat energy will need to go into the electrons to match observations (e.g. Ball, Narayan \& Quataert 2001). Detailed models are awaited.

This work was supported in part by NSF grant AST 9820686, RFBR grant 00-02-16135, and Swedish NFR grant. EQ acknowledges support provided by NASA through Chandra Fellowship grant number PF9-10008 awarded by the Chandra X-ray Center, which is operated by the Smithsonian Astrophysical Observatory for NASA under contract NAS8-39073. 


\section{REFERENCES}

Ball, G., Narayan, R., \& Quataert, E. 2001, ApJ,

Begelman, M. C., \& Meier, D. L. 1982, ApJ, 253, 873

Chen, X., Abramowicz, M. \& Lasota, J.-P., 1997, ApJ, 476, 61

Esin, A.A., McClintock, J.E., Drake, J.J., Garcia, M.R., Haswell, C.A., Hynes, R.I., \& Muno, M.P. 2001, ApJ, in press astro-ph/0103044

Esin, A.A., McClintock, J.E., \& Narayan, R. 1997, ApJ, 489, 865

Esin, A.A., Narayan, R., Cui, W., Grove, J.E., \& Zhang, S.-N. 1998, ApJ, 505, 854

Gilham, S. 1981, MNRAS, 195, 755

Hawley, J. F., Balbus, S. A., \& Stone, J. M. 2001, ApJ, submitted (astro-ph/0103522)

Igumenshchev, I. V. 2000, MNRAS, 314, 54

Igumenshchev, I. V., \& Abramowicz, M. A. 1999, MNRAS, 303, 309

Igumenshchev, I. V., \& Abramowicz, M. A. 2000, ApJS, 130, 463

Igumenshchev, I. V., Abramowicz, M. A., \& Narayan, R. 2000, ApJ, 537, L271

Igumenshchev, I. V., \& Narayan, R. 2001, ApJ, submitted astro-ph/0105365

Kato, S., Fukue, J., \& Mineshige, S. 1998, Black-Hole Accretion Disks (Kyoto: Kyoto Univ. Press)

Machida, M., Matsumoto, R., \& Mineshige, S. 2001, PASJ, 53, L1

Melia, F. 1992, ApJ, 387, L25

Narayan, R. 1992, ApJ, 394, 261

Narayan, R. 1996, ApJ, 462, 136

Narayan, R., \& Yi, I. 1994, ApJ, 428, L13

Narayan, R., \& Yi, I. 1995, ApJ, 444, 231

Narayan, R., Kato, S., \& Honma, F., 1997, ApJ, 476, 49
Narayan, R., Mahadevan, R., \& Quataert, E. 1998, in The Theory of Black Hole Accretion Disks, ed. M. A. Abramowicz, G. Bjornsson, \& J. E. Pringle (Cambridge: Cambridge Univ. Press), 148

Narayan, R., Igumenshchev, I. V., \& Abramowicz, M. A. 2000, ApJ, 539, 798

Paczyǹski, B. \& Wiita, P., J., 1980, A\&A, 88, 23

Popham, R., \& Narayan, R. 1992, ApJ, 394, 255

Quataert, E., \& Gruzinov, A. 2000, ApJ, 539, 809

Quataert, E., \& Narayan, R. 1999, ApJ, 520, 298

Ryu, D., \& Goodman, J. 1992, ApJ, 388, 438

Stone, J. M., \& Balbus, S. A. 1996, ApJ, 464, 364

Stone, J. M., Pringle, J. E., \& Begelman, M. C. 2001, MNRAS, 310, 1002

This 2-column preprint was prepared with the AAS LATEX macros $\mathrm{v} 4.0$. 


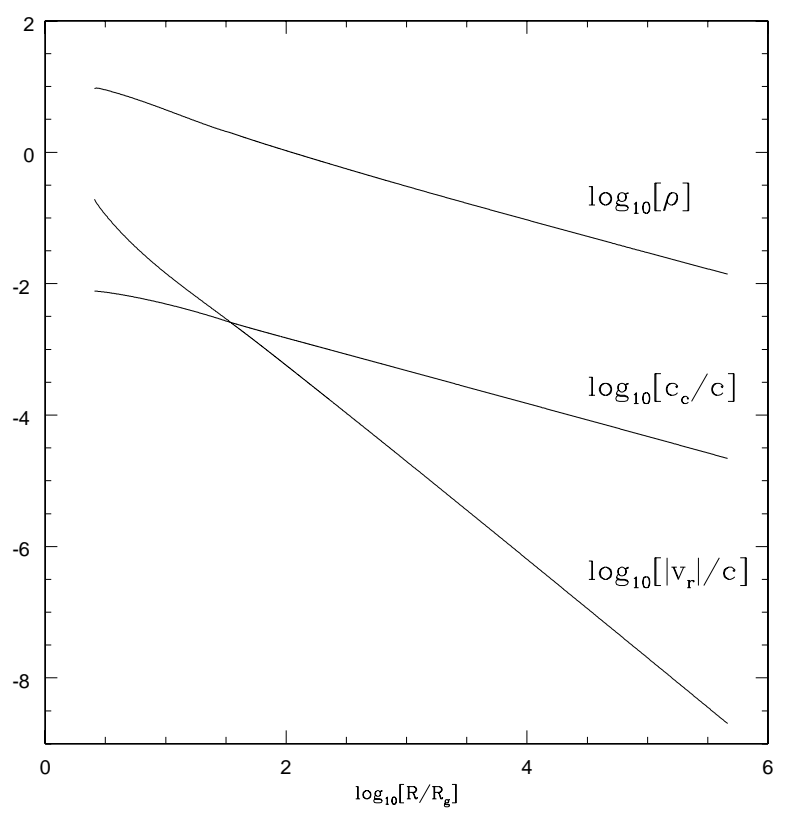

Fig. 1. - Global CDAF model with $\alpha=0.03$ and $\gamma=1.5$. The density profile $\rho$ is in arbitrary units. Inside $R=R_{A C} \approx 35 R_{g}$ the inflow speed exceeds the local convective velocity $\left(c_{c}\right)$ and energy transport in the flow is dominated by inward advection. At larger radii, the flow is roughly static $\left(\left|v_{r}\right| \ll c_{c}\right)$ and energy is transported outward by convection. 


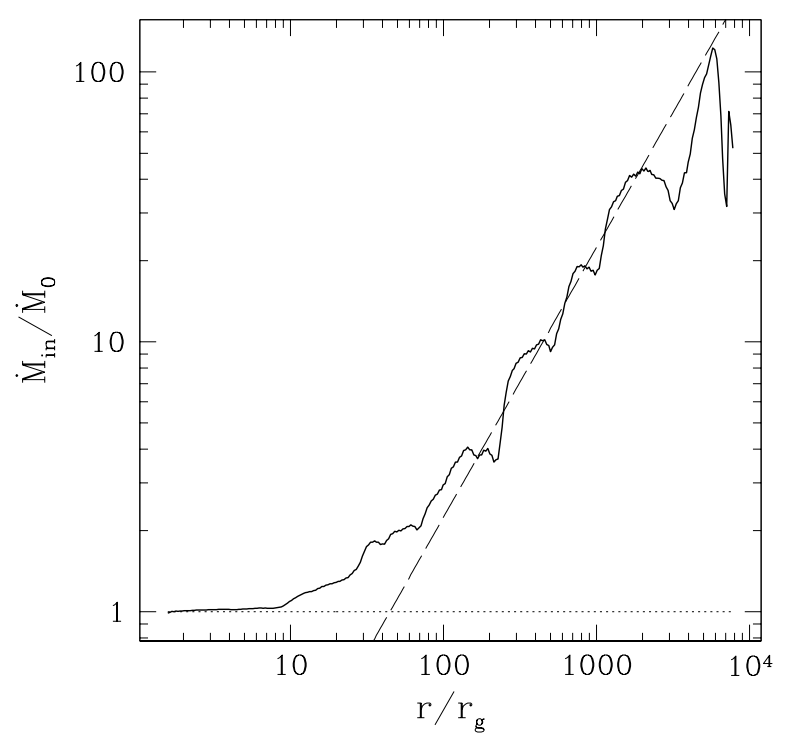

Fig. 2.- Instantaneous mass inflow rate $\dot{M}_{\text {in }}$ (solid line) as a function of radius $R$ in a $2 \mathrm{D}$ hydrodynamical model of a radiatively inefficient accretion flow with $\gamma=5 / 3$ and $\alpha=0.01$. The values of $\dot{M}_{i n}$ are calculated by adding all the inflowing gas elements at a given $R . \dot{M}_{i n}$ is normalized to the net accretion rate $\dot{M}_{0}$. Except near the outer boundary, $R \gtrsim 2 \times 10^{3} R_{g}$ and in the inner region, $R \lesssim 10^{2} R_{g}$, the profile $\dot{M}_{i n}(R)$ shows good agreement with the prediction of the self-similar solution for convectiondominated accretion flows, $\dot{M}_{i n} \propto R$, shown by the long-dashed line. Deviation of $\dot{M}_{i n}$ from the selfsimilar CDAF scaling in the inner region is due to the strong inward advection of internal energy, as discussed in the text. 


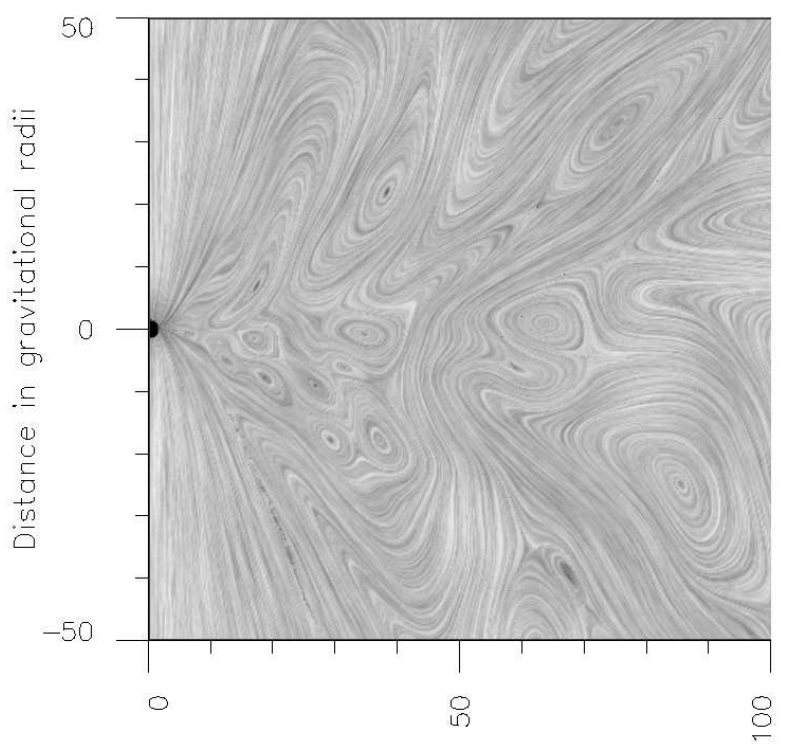

Fig. 3.- Snapshot of streamlines from a 2D hydrodynamical model of a radiatively inefficient accretion flow with $\alpha=0.01$ and $\gamma=5 / 3$. A projection of streamlines on the meridional cross-section is shown, with the black hole located at the origin. The flow pattern is highly time dependent and consists of numerous temporal vortices of different spatial scales. In the innermost region of the flow the energy balance is dominated by inward advection of internal energy. Convective blobs originate in this region and move toward the outer convection-dominated region at radii $\gtrsim 10^{2} R_{g}$. 